Proc. Indian Acad. Sci. (Math. Sci.) Vol. 114, No. 1, February 2003, pp. 79-96.

Printed in India

\title{
Inverse solutions for a second-grade fluid for porous medium channel and Hall current effects
}

\author{
MUHAMMAD R MOHYUDDIN ${ }^{1,2}$ and EHSAN ELLAHI ASHRAF ${ }^{3}$ \\ ${ }^{1}$ Corresponding author: Department of Mathematics, Quaid-i-Azam University, 45320, \\ Islamabad 44000, Pakistan \\ ${ }^{2}$ Present address: Department of Condensed Matter Physics, Main Building, \\ Strada Costeira 11, 34014, ICTP, Trieste, Italy \\ ${ }^{3}$ College of Aeronautical Engineering, National University of Sciences and \\ Technology, PAF Academy, Risalpur 24090, Pakistan \\ E-mail: ${ }^{1}$ m_raheel@yahoo.com; ${ }^{2}$ mmohyudd@ictp.trieste.it
}

MS received 29 August 2003; revised 19 December 2003

\begin{abstract}
Assuming certain forms of the stream function inverse solutions of an incompressible viscoelastic fluid for a porous medium channel in the presence of Hall currents are obtained. Expressions for streamlines, velocity components and pressure fields are described in each case and are compared with the known viscous and secondgrade cases.
\end{abstract}

Keywords. Second-grade fluid; exact solutions; Hall effects; porous medium.

\section{Introduction}

In recent years the theoretical study of MHD channel flows has been a subject of great interest due to its widespread applications in designing cooling systems with liquid metals, petroleum industry, purification of crude oil, polymer technology, centrifugal separation of matter from fluid, MHD generators, pumps, accelerators and flow meters. The results of these investigations are not applicable to the flow of ionized gases. In an ionized gas where the density is low and/or the magnetic field is very strong, the conductivity normal to the free spiraling of electrons and ions about the magnetic lines of force before suffering collisions; also, a current is induced in a direction normal to both the electric and magnetic fields. The phenomena, well-known in the literature, is called the Hall effect. The study of magnetohydrodynamic flows with Hall currents has important engineering applications in problems of magnetohydrodynamics generators and of Hall accelerators as well as in flight magnetohydrodynamics [910 11:18 20 2123|24].

An understanding of the dynamics of fluids in porous media has practical interest in such disparate fields as petroleum engineering and ground water hydrology, with applications ranging from hydrocarbon migration in reservoirs via packed-bed chemical reactors to agricultural drainage and irrigation. In the widely used continuum approach to transport processes in porous media, the differential equation governing the macroscopic fluid motion is based on the experimentally established Darcy's law [2], which accounts for the drag exerted by the porous medium. Brinkman [5] studied Darcy's law by adding to it a viscous term in order to account for the vorticity diffusion caused by the boundary resistance, whereas the combined influence of inertia and viscous effects on the flow and 
heat transfer in the vicinity of an impermeable plane surface was discussed by Vafai and Tien [28] and Kaviany [13]. Kaviany's analysis was for steady-state flow, the oscillatory motion was studied by Khodadadi [14]15] and transient fluid motion in a porous medium channel was discussed by Anderson and Holmedal [1].

The governing equations that describe the flow of a Newtonian fluid is the NavierStokes equations. These equations are non-linear partial differential equations and known exact solutions are few in number. Exact solutions are very important not only because they are solutions of some fundamental flows but also because they serve as accuracy checks for experimental, numerical and asymptotic methods. Since the equations of motion of non-Newtonian fluids are more complicated and non-linear than the NavierStokes equations, so the inverse methods described by Nemenyi [17] have become attractive. In these methods, solutions are found by assuming certain physical or geometrical properties of the flow field. Kaloni and Huschilt [12], Siddiqui and Kaloni [25], Siddiqui [26], Benharbit and Siddiqui [3], Labropulu [16] and Siddiqui et al [27] used this method to study the flow problems of a second-grade fluid.

In this work, we discuss the effects of Hall currents on the steady flow of an electrically conducting second-grade fluid in a porous medium channel. For such a fluid equations are modeled for a grade of fluid two and are solved by assuming certain form of the stream function. The graphs are plotted explicitly in the functional form to see the behaviour of the flow field.

The paper is arranged in the following fashion: In $\S 2$, governing equations and formulation of the problem are given. Section 3 consists of two parts. First part is the generalization of Siddiqui's [26] work and the second part deals with some special flows called as Riabounchinsky type flows and finally, concluding remarks are given in $\S 4$. Stream function, velocity components and the pressure fields are derived in each case. Moreover, the streamlines are plotted in each case to see the flow behaviour.

\section{Governing equations}

The constitutive equation of an incompressible fluid of second-grade is of the form [22]

$$
\mathbf{T}=-p \mathbf{I}+\mu \mathbf{A}_{1}+\alpha_{1} \mathbf{A}_{2}+\alpha_{2} \mathbf{A}_{1}^{2},
$$

where $\mathbf{T}$ is the Cauchy stress tensor, $-p \mathbf{I}$ denotes the indeterminate spherical stress and $\mu, \alpha_{1}$ and $\alpha_{2}$ are measurable material constants. They denote, respectively, the viscosity, elasticity and cross-viscosity. These material constants can be determined from viscometric flows for any real fluid. $\mathbf{A}_{1}$ and $\mathbf{A}_{2}$ are Rivlin-Ericksen tensors [22] and they denote, respectively, the rate of strain and acceleration. $\mathbf{A}_{1}$ and $\mathbf{A}_{2}$ are defined by

$$
\begin{aligned}
& \mathbf{A}_{1}=(\operatorname{grad} \mathbf{V})+(\operatorname{grad} \mathbf{V})^{\top}, \\
& \mathbf{A}_{2}=\frac{\mathrm{d} \mathbf{A}_{1}}{\mathrm{~d} t}+\mathbf{A}_{1}(\operatorname{grad} \mathbf{V})+(\operatorname{grad} \mathbf{V})^{\top} \mathbf{A}_{1} .
\end{aligned}
$$

Here $\mathbf{V}$ is the velocity, grad the gradient operator, $T$ the transpose, and $\mathrm{d} / \mathrm{d} t$ the material time derivative.

The basic equations governing the motion of an incompressible fluid are

$$
\begin{aligned}
& \nabla \cdot \mathbf{V}=0, \\
& \rho \frac{\mathrm{d} \mathbf{V}}{\mathrm{d} t}=\mathbf{J} \times \mathbf{B}+\operatorname{div} \mathbf{T}-\frac{\mu}{K} \mathbf{V},
\end{aligned}
$$




$$
\nabla \cdot \mathbf{B}=0, \nabla \times \mathbf{B}=\mu_{m} \mathbf{J}, \nabla \times \mathbf{E}=0
$$

where $\rho$ is the density, $\mathbf{J}$ the current density, B the total magnetic field, $\mu_{m}$ the magnetic permeability, $\mathbf{E}$ the total electric field current and $K$ the permeability of the porous medium. Making reference to Cowling [6], when the strength of the magnetic field is very large, the generalized Ohm's law is modified to include the Hall current so that

$$
\mathbf{J}+\frac{\omega_{e} \tau_{e}}{\mathbf{B}_{0}}(\mathbf{J} \times \mathbf{B})=\sigma\left[\mathbf{E}+\mathbf{V} \times \mathbf{B}+\frac{1}{e n_{e}} \nabla p_{e}\right]
$$

in which $\omega_{e}$ is the cyclotron frequency, $\tau_{e}$ the electron collision time, $\sigma$ the electrical conductivity, $e$ the electron charge and $p_{e}$ the electron pressure. The ion-slip and thermoelectric effects are not included in (2.7). Further, it is assumed that $\omega_{e} \tau_{e} \sim O 1$ and $\omega_{i} \tau_{i} \ll 1$, where $\omega_{i}$ and $\tau_{i}$ are the cyclotron frequency and collision time for ions respectively.

Inserting (2.1) in (2.5) and making use of (2.2), (2.3), (2.6) and (2.7) we obtain the following vector equation

$$
\begin{aligned}
\operatorname{grad}[ & \left.\frac{1}{2} \rho|\mathbf{V}|^{2}+p-\alpha_{1}\left(\mathbf{V} \cdot \nabla^{2} \mathbf{V}+\frac{1}{4}\left|\mathbf{A}_{1}\right|^{2}\right)\right]+\rho\left[\mathbf{V}_{t}-\mathbf{V} \times(\nabla \times \mathbf{V})\right] \\
= & \mu \nabla^{2} \mathbf{V}+\alpha_{1}\left[\nabla^{2} \mathbf{V}_{t}+\nabla^{2}(\nabla \times \mathbf{V}) \times \mathbf{V}\right] \\
& +\left(\alpha_{1}+\alpha_{2}\right) \operatorname{div} \mathbf{A}_{1}^{2}-\frac{\sigma \mathbf{B}_{0}^{2}(1+i \varphi)}{1+\varphi^{2}}-\frac{\mu}{K} \mathbf{V}
\end{aligned}
$$

in which $\nabla^{2}$ is the Laplacian operator, $\mathbf{V}_{t}=\partial \mathbf{V} / \partial t$, and $\left|\mathbf{A}_{1}\right|$ is the usual norm of matrix $\mathbf{A}_{1}$. If this model is required to be compatible with thermodynamics, then the material constants must meet the restrictions [78]

$$
\mu \geq 0, \alpha_{1} \geq 0, \alpha_{1}+\alpha_{2}=0
$$

On the other hand, experimental results of tested fluids of second-grade showed that $\alpha_{1}<0$ and $\alpha_{1}+\alpha_{2} \neq 0$ which contradicts the above conditions and imply that such fluids are unstable. This controversy is discussed in detail in [19]. However, in this paper we will discuss both cases, $\alpha_{1} \geq 0$ and $\alpha_{1}<0$.

The velocity field for the problem under consideration is of the following form

$$
\mathbf{V}(x, y, t)=[u(x, y, t), v(x, y, t), 0]
$$

where $u$ and $v$ are velocity components in the $x$ and $y$ directions, respectively.

Inserting (2.10) in (2.4) and (2.8) and making use of the assumption (2.9) we obtain the following equations

$$
\begin{aligned}
\frac{\partial u}{\partial x}+\frac{\partial v}{\partial y}=0, & \\
\frac{\partial \widehat{p}}{\partial x}+\rho\left[\frac{\partial u}{\partial t}-v \omega\right]= & \left(\mu+\alpha_{1} \frac{\partial}{\partial t}\right) \nabla^{2} u-\alpha_{1} v \nabla^{2} \omega \\
& -\frac{\sigma \mathbf{B}_{0}^{2}(1+i \varphi)}{1+\varphi^{2}} u-\frac{\mu}{K} u
\end{aligned}
$$




$$
\begin{aligned}
\frac{\partial \widehat{p}}{\partial y}+\rho\left[\frac{\partial v}{\partial t}+u \omega\right]= & \left(\mu+\alpha_{1} \frac{\partial}{\partial t}\right) \nabla^{2} v+\alpha_{1} u \nabla^{2} \omega \\
& -\frac{\sigma \mathbf{B}_{0}^{2}(1+i \varphi)}{1+\varphi^{2}} v-\frac{\mu}{K} v
\end{aligned}
$$

where $\varphi=\omega_{e} \tau_{e}$ is the Hall parameter and

$$
\begin{aligned}
& \omega=\frac{\partial v}{\partial x}-\frac{\partial u}{\partial y} \\
& \widehat{p}=p+\frac{1}{2} \rho\left(u^{2}+v^{2}\right)-\alpha_{1}\left[u \nabla^{2} u+v \nabla^{2} v+\frac{1}{4}\left|\mathbf{A}_{1}^{2}\right|\right] \\
& \left|\mathbf{A}_{1}^{2}\right|=4\left(\frac{\partial u}{\partial x}\right)^{2}+4\left(\frac{\partial v}{\partial y}\right)^{2}+2\left(\frac{\partial u}{\partial y}+\frac{\partial v}{\partial x}\right)^{2}
\end{aligned}
$$

Remark 1. On setting $\alpha_{1}=0, K \rightarrow \infty$ and neglecting Hall effects in (2.12) and (2.13) we recover the equations for viscous fluid, on taking $K \rightarrow \infty$ and neglecting Hall effects we obtain the case [26] and on taking $\alpha_{1}=0$ and $\varphi=0$ in Hall effects we obtain the Brinkman model for porous medium.

Equations (2.11)-(2.13) are three partial differential equations for three unknown functions $u, v$ and $\widehat{p}$ of the variables $(x, y)$. Once the velocity field is determined, the pressure field (2.15) can be calculated by integrating (2.12) and (2.13). Note that the equation for the component $w$ is identically zero.

Eliminating pressure in (2.12) and (2.13), by applying the integrability condition $\partial^{2} \widehat{p} / \partial x \partial y=\partial^{2} \hat{p} / \partial y \partial x$, we get the compatibility equation

$$
\begin{aligned}
\rho\left[\frac{\partial \omega}{\partial t}+\left(u \frac{\partial}{\partial x}+v \frac{\partial}{\partial y}\right) \omega\right]= & \left(\mu+\alpha_{1} \frac{\partial}{\partial t}\right) \nabla^{2} \omega-\frac{\sigma \mathbf{B}_{0}^{2}(1+i \varphi)}{1+\varphi^{2}} \omega \\
& -\frac{\mu}{K} \omega+\alpha_{1}\left[\left(u \frac{\partial}{\partial x}+v \frac{\partial}{\partial y}\right) \nabla^{2} \omega\right] .
\end{aligned}
$$

Let us consider the Stokes stream function:

$$
u=\frac{\partial \psi}{\partial y}, v=-\frac{\partial \psi}{\partial x}
$$

where $\psi(x, y)$ is the stream function. We see that the continuity equation (2.11) is satisfied identically and (2.18) in (2.17) yields the following equation

$$
\begin{aligned}
\rho\left[\frac{\partial}{\partial t} \nabla^{2} \psi-\left\{\psi, \nabla^{2} \psi\right\}\right]= & \left(\mu+\alpha_{1} \frac{\partial}{\partial t}\right) \nabla^{4} \psi-\alpha_{1}\left[\left\{\psi, \nabla^{4} \psi\right\}\right] \\
& -\frac{\sigma \mathbf{B}_{0}^{2}(1+i \varphi)}{1+\varphi^{2}} \nabla^{2} \psi-\frac{\mu}{K} \nabla^{2} \psi
\end{aligned}
$$

in which

$$
\nabla^{4}=\nabla^{2} \cdot \nabla^{2}, \omega=-\nabla^{2} \psi
$$


and

$$
\left\{\psi, \nabla^{2} \psi\right\}=\frac{\partial \psi}{\partial x} \frac{\partial\left(\nabla^{2} \psi\right)}{\partial y}-\frac{\partial \psi}{\partial y} \frac{\partial\left(\nabla^{2} \psi\right)}{\partial x}
$$

is the Poisson bracket.

Remark 2. The equation (2.19), for $K \rightarrow \infty$ and in the absence of Hall effects reduces to [26].

\section{Solutions of some special types}

\subsection{Solution of the type $\psi(x, y)=\xi(x)+\eta(y)$}

We consider the plane steady flow and examine the solution of (2.19) of the form

$$
\psi(x, y)=\xi(x)+\eta(y),
$$

where $\xi$ and $\eta$ are arbitrary functions of the variables $x$ and $y$ respectively. Substituting (3.1.1) in (2.19) we obtain the following equation

$$
\begin{aligned}
\rho\left[\eta^{\prime}(y) \xi^{\prime \prime \prime}(x)-\xi^{\prime}(x) \eta^{\prime \prime \prime}(y)\right]= & \mu\left[\xi^{\mathrm{IV}}(x)+\eta^{\mathrm{IV}}(y)\right] \\
& +\alpha_{1}\left[\eta^{\prime}(y) \xi^{\mathrm{V}}(x)-\xi^{\prime}(x) \eta^{\mathrm{V}}(y)\right] \\
& +\left(\frac{\sigma \mathbf{B}_{0}^{2}(1+i \varphi)}{1+\varphi^{2}}+\frac{\mu}{K}\right)\left[\xi^{\prime \prime}(x)+\eta^{\prime \prime}(y)\right],
\end{aligned}
$$

in which IV and V in the superscript indicates the fourth and fifth derivatives.

We see that (3.1.2) is highly non-linear and its solution in the present form is not easy to obtain. In order to find its solution we assume the following

$$
\begin{aligned}
& \xi(x)=A x+B \mathrm{e}^{a x}, \\
& \eta(y)=C y+D \mathrm{e}^{b y}
\end{aligned}
$$

and obtain the following equation

$$
\begin{aligned}
\rho[- & \left.A b^{3} D \mathrm{e}^{b y}+a^{3} B C \mathrm{e}^{a x}-a b^{3} B D \mathrm{e}^{a x+b y}+a^{3} b B D \mathrm{e}^{a x+b y}\right] \\
= & \mu\left[a^{4} B \mathrm{e}^{a x}+b^{4} D \mathrm{e}^{b y}\right]+\alpha_{1}\left[\begin{array}{c}
-A b^{5} D \mathrm{e}^{b y}+a^{5} B C \mathrm{e}^{a x} \\
-a b^{5} B D \mathrm{e}^{a x+b y}+a^{5} b B D \mathrm{e}^{a x+b y}
\end{array}\right] \\
& -\left(\frac{\sigma \mathbf{B}_{0}^{2}(1+i \varphi)}{1+\varphi^{2}}+\frac{\mu}{K}\right)\left[a^{2} B \mathrm{e}^{a x}+b^{2} D \mathrm{e}^{b y}\right]
\end{aligned}
$$

where $A, B, C, D, a$ and $b$ are arbitrary constants.

The following three equations are obtained from (3.1.5)

$$
\begin{aligned}
& \rho a C=\mu a^{2}+\alpha_{1} a^{3} C-H-\frac{\mu}{K}, \\
& -\rho b A=\mu b^{2}-\alpha_{1} b^{3} A-H-\frac{\mu}{K}, \\
& \left(b^{2}-a^{2}\right)\left[\rho-\alpha_{1}\left(a^{2}+b^{2}\right)\right]=0 .
\end{aligned}
$$


From (3.1.6) and (3.1.7) we easily obtain the values of $A$ and $C$, i.e.

$$
\begin{aligned}
& A=-\frac{1}{\rho-\alpha_{1} b^{2}}\left[\mu b-\frac{H}{b}-\frac{\mu}{K b}\right], \\
& C=\frac{1}{\rho-\alpha_{1} a^{2}}\left[\mu a-\frac{H}{a}-\frac{\mu}{K a}\right],
\end{aligned}
$$

where

$$
H=\frac{\sigma \mathbf{B}_{0}^{2}(1+i \varphi)}{1+\varphi^{2}}
$$

and (3.1.8) is satisfied if either

$$
b^{2}-a^{2}=0
$$

or

$$
\rho=\alpha_{1}\left(a^{2}+b^{2}\right)
$$

We have three different cases which we discuss separately as follows:

Case 1. $b=a, \rho \neq \alpha_{1}\left(a^{2}+b^{2}\right)$

The stream function given by (3.1.1), after using (3.1.3), (3.1.4), (3.1.9), and (3.1.10) becomes

$$
\psi(x, y)=\frac{(y-x)}{\rho-\alpha_{1} a^{2}}\left[\mu a-\frac{H}{a}-\frac{\mu}{K a}\right]+B \mathrm{e}^{a x}+D \mathrm{e}^{a y}
$$

and from (2.18) the velocity components take the following form

$$
\begin{aligned}
& u=\frac{1}{\rho-\alpha_{1} a^{2}}\left[\mu a-\frac{H}{a}-\frac{\mu}{K a}\right]+D a \mathrm{e}^{a y}, \\
& v=\frac{1}{\rho-\alpha_{1} a^{2}}\left[\mu a-\frac{H}{a}-\frac{\mu}{K a}\right]-B a \mathrm{e}^{a x} .
\end{aligned}
$$

In order to find the pressure field (2.15) we substitute the velocity components $(3.1 .14)$ and (3.1.15) in (2.12) and (2.13) and then integrate the resulting equations to obtain

$$
\begin{aligned}
p= & p_{0}-\rho \bar{a}^{2}-\mu B a^{3} y \mathrm{e}^{a x}+\left(\rho-\alpha_{1} a^{2}\right)\left[a^{2} B y \mathrm{e}^{a x}+a^{2} D B \mathrm{e}^{a(x+y)}\right] \\
& +\alpha_{1}\left[B^{2} a^{4} \mathrm{e}^{2 a x}+D^{2} a^{4} \mathrm{e}^{2 a y}-D B a^{4} \mathrm{e}^{a(x+y)}\right],
\end{aligned}
$$

where

$$
\bar{a}=\frac{1}{\rho-\alpha_{1} a^{2}}\left[\mu a-\frac{H}{a}-\frac{\mu}{K a}\right],
$$

whereas the streamline for $\psi=\Omega_{1}$ is given by the following functional form

$$
y=\frac{-B \mathrm{e}^{a x}+x \varepsilon+\Omega_{1}}{\varepsilon}-\frac{1}{a} \text { Product } \log \left[\frac{D a}{\varepsilon} \mathrm{e}^{a\left(-B \mathrm{e}^{a x}+x \varepsilon+\Omega_{1}\right) / \varepsilon}\right],
$$




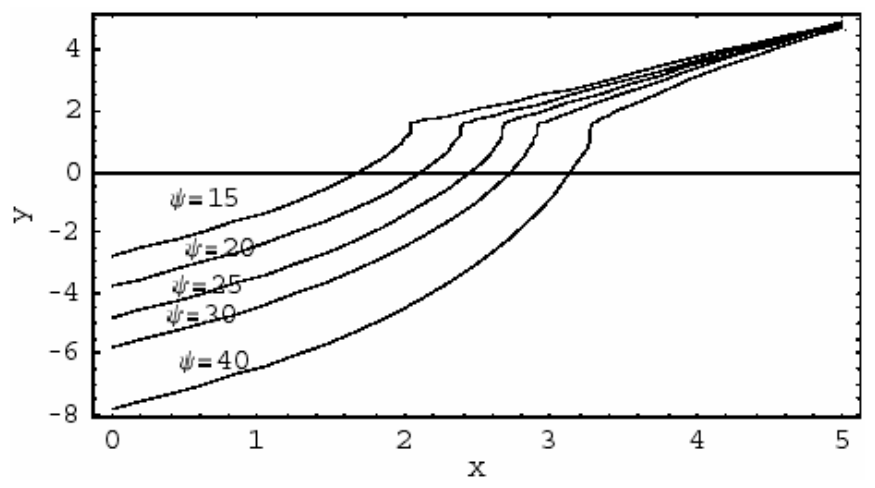

Figure 1. Streamline flow pattern for $\psi(x, y)=\frac{(y-x)}{\rho-\alpha_{1} a^{2}}\left[\mu a-\frac{H}{a}-\frac{\mu}{K a}\right]+B \mathrm{e}^{a x}$ $+D \mathrm{e}^{a y}$.

where

$$
\varepsilon=\frac{1}{1-\Lambda a^{2}}\left[v a-\frac{\chi}{a}-\frac{v}{K a}\right]
$$

with $v=\mu / \rho$ as the kinematic viscosity, $\Lambda=\alpha_{1} / \rho$ is the second-grade parameter $\chi=$ $N(1+i \varphi) /\left(1+\varphi^{2}\right), N=\sigma \mathbf{B}_{0}^{2} / \rho$ is the MHD parameter and $\varphi=\omega_{e} \tau_{e}$ is the Hall parameter.

Streamlines are shown in figure 1 for $B=D=a=1, \mu / \rho=0.5, \alpha_{1} / \rho=0.1, K=0.1$, $N=0, \omega_{e} \tau_{e}=0.1$ and $\psi=15,20,25,30,40$.

Case 2. $b=-a, \rho \neq \alpha_{1}\left(a^{2}+b^{2}\right)$

The expressions for $\psi, u, v$, and $p$ are

$$
\begin{aligned}
& \psi(x, y)=\frac{1}{\rho-\alpha_{1} a^{2}}\left[\mu a-\frac{H}{a}-\frac{\mu}{K a}\right](y+x)+B \mathrm{e}^{a x}+D \mathrm{e}^{-a y}, \\
& u=\frac{1}{\rho-\alpha_{1} a^{2}}\left[\mu a-\frac{H}{a}-\frac{\mu}{K a}\right]-D a \mathrm{e}^{-a y}, \\
& v=-\frac{1}{\rho-\alpha_{1} a^{2}}\left[\mu a-\frac{H}{a}-\frac{\mu}{K a}\right]-B a \mathrm{e}^{a x}, \\
& p=p_{0}-\rho \bar{a}^{2}-\mu B a^{3} y \mathrm{e}^{a x}+\left(\rho-\alpha_{1} a^{2}\right)\left[a^{2} B y \mathrm{e}^{a x}+a^{2} D B \mathrm{e}^{a(x-y)}\right] \\
& +\alpha_{1}\left[B^{2} a^{4} \mathrm{e}^{2 a x}+D^{2} a^{4} \mathrm{e}^{-2 a y}-D B a^{4} \mathrm{e}^{a(x-y)}\right]
\end{aligned}
$$

and the functional form of streamline for $\psi=\Omega_{2}$ is given by

$$
y=\frac{-B \mathrm{e}^{a x}-x \varepsilon+\Omega_{2}}{\varepsilon}+\frac{1}{a} \text { Product Log }\left[-\frac{D a}{\varepsilon} \mathrm{e}^{a\left(B \mathrm{e}^{a x}+x \varepsilon-\Omega_{2}\right) / \varepsilon}\right] .
$$

Streamlines are drawn in figure 2 for $B=D=a=1, \mu / \rho=0.5, \alpha_{1} / \rho=0.1, K=0.1$, $N=0.5, \omega_{e} \tau_{e}=1$ and $\psi=15,20,25,30,40$. 


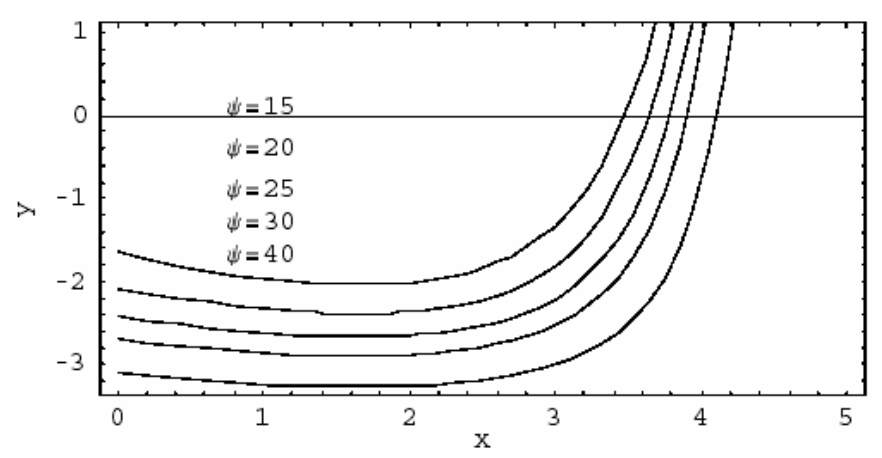

Figure 2. Streamline flow pattern for $\psi(x, y)=\frac{1}{\rho-\alpha_{1} a^{2}}\left[\mu a-\frac{H}{a}-\frac{\mu}{K a}\right](y+x)$ $+B \mathrm{e}^{a x}+D \mathrm{e}^{-a y}$.

Case 3. $b^{2}-a^{2} \neq 0$

We must have

$$
\rho=\alpha_{1}\left(a^{2}+b^{2}\right)
$$

and the expressions for $\psi, u, v$, and $p$ are of the following form

$$
\begin{aligned}
& \psi(x, y)=-\frac{x}{\rho-\alpha_{1} b^{2}}\left[\mu b-\frac{H}{b}-\frac{\mu}{K b}\right] \\
& +\frac{y}{\rho-\alpha_{1} a^{2}}\left[\mu a-\frac{H}{a}-\frac{\mu}{K a}\right]+B \mathrm{e}^{a x}+D \mathrm{e}^{b y}, \\
& u=\frac{1}{\rho-\alpha_{1} a^{2}}\left[\mu a-\frac{H}{a}-\frac{\mu}{K a}\right]+D b \mathrm{e}^{b y}, \\
& v=\frac{1}{\rho-\alpha_{1} b^{2}}\left[\mu b-\frac{H}{b}-\frac{\mu}{K b}\right]-B a \mathrm{e}^{a x}, \\
& p=p_{0}-\frac{1}{2} \rho\left[\bar{a}^{2}+\bar{b}^{2}\right]-\mu B a^{3} y \mathrm{e}^{a x} \\
& +\left(\rho-\alpha_{1} a^{2}\right)\left[a^{2} B y \mathrm{e}^{a x}+a^{2} D B \mathrm{e}^{a x+b y}\right] \\
& +\alpha_{1}\left[B^{2} a^{4} \mathrm{e}^{2 a x}+D^{2} a^{4} \mathrm{e}^{2 b y}-D B a^{4} b^{2} \mathrm{e}^{a x+b y}\right],
\end{aligned}
$$

where

$$
\bar{a}=\frac{1}{\rho-\alpha_{1} a^{2}}\left[\mu a-\frac{H}{a}-\frac{\mu}{K a}\right], \quad \bar{b}=\frac{1}{\rho-\alpha_{1} b^{2}}\left[\mu b-\frac{H}{b}-\frac{\mu}{K b}\right],
$$

whereas the functional form in this case for $\psi=\Omega_{3}$ is

$$
y=-\frac{B \mathrm{e}^{a x}-x \delta-\Omega_{3}}{\varepsilon}-\frac{1}{b} \text { Product Log }\left[\frac{D b}{\varepsilon} \mathrm{e}^{-b\left(B \mathrm{e}^{a x}-x \delta-\Omega_{3}\right) / \varepsilon}\right],
$$

where

$$
\delta=\frac{1}{1-\Lambda b^{2}}\left[v b-\frac{\chi}{b}-\frac{v}{K b}\right]
$$


Streamlines for $B=D=a=1, \mu / \rho=0.5, b=-0.5, K=2,2.1, \alpha_{1} / \rho=0.5,-0.7, N=$ $0.5, \omega_{e} \tau_{e}=1$ and $\psi=15,20,25,30,40$ are depicted in figures 3 , 4 , whereas figure 5 is given for $\alpha_{1} / \rho=-0.5$.

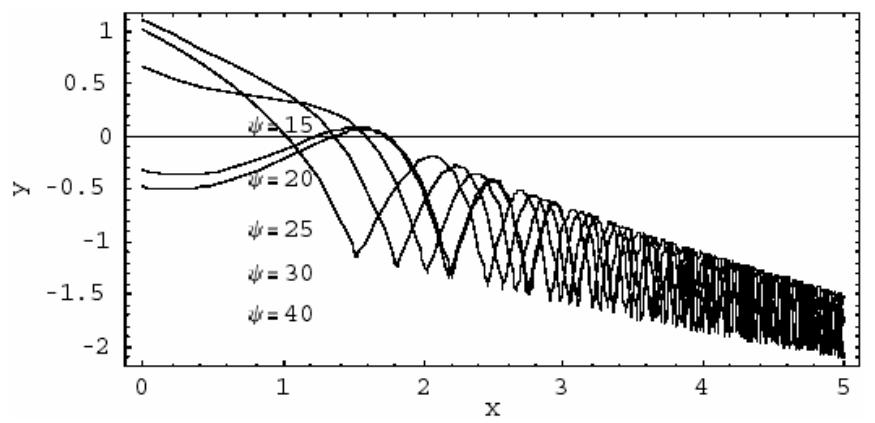

Figure 3. $\psi(x, y)=-x \bar{a}+y \bar{b}+B \mathrm{e}^{a x}+D \mathrm{e}^{b y}$.

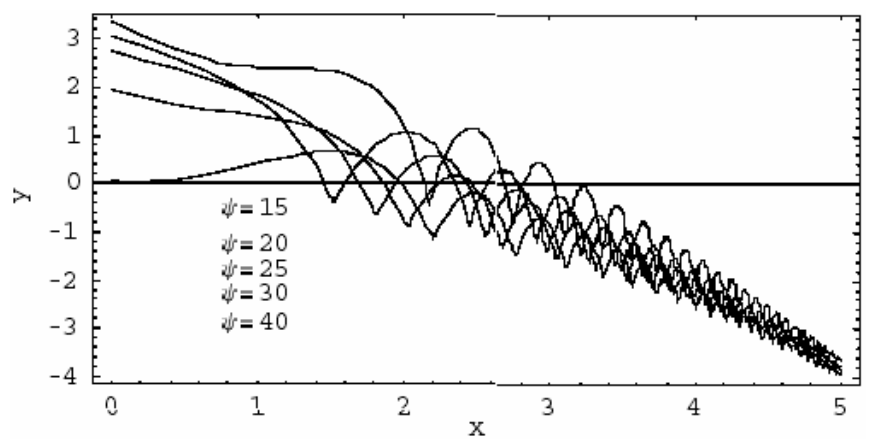

Figure 4. Streamline flow pattern for $\psi(x, y)=-x \bar{a}+y \bar{b}+B \mathrm{e}^{a x}+D \mathrm{e}^{b y}$.

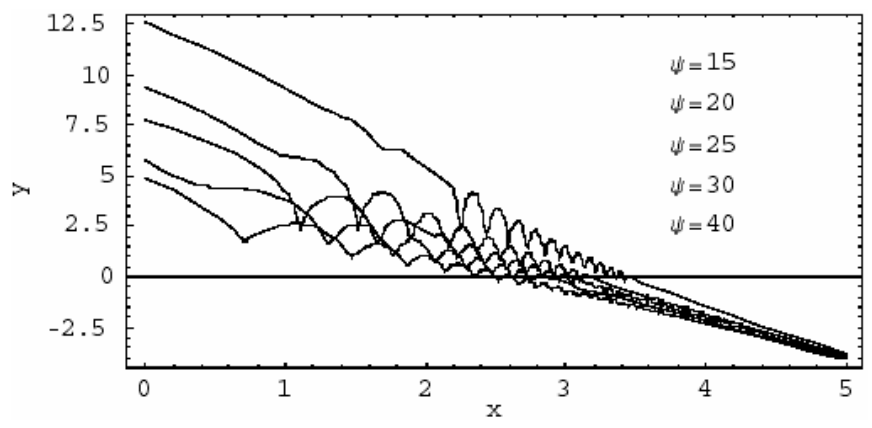

Figure 5. Streamline flow pattern for negative second-grade parameter. 
The alternate forms of (3.1.23) may be written as

$$
\begin{aligned}
\psi(x, y)= & -\frac{x}{\rho-\alpha_{1} b^{2}}\left[\mu b-\frac{H}{b}-\frac{\mu}{K b}\right] \\
& +\frac{y}{\alpha_{1} b^{2}}\left[\mu a-\frac{H}{a}-\frac{\mu}{K a}\right]+B \mathrm{e}^{a x}+D \mathrm{e}^{b y}, \\
\psi(x, y)= & -\frac{x}{\alpha_{1} a^{2}}\left[\mu b-\frac{H}{b}-\frac{\mu}{K b}\right] \\
& +\frac{y}{\rho-\alpha_{1} a^{2}}\left[\mu a-\frac{H}{a}-\frac{\mu}{K a}\right]+B \mathrm{e}^{a x}+D \mathrm{e}^{b y} .
\end{aligned}
$$

Remark 3. The solution (3.1.16) with $H=0, K \rightarrow \infty$ and $\alpha_{1}=0$ gives the Berker's solution [4] and the Siddiqui's solutions [26] can readily be recovered as a special case by taking $H=0$ and $K \rightarrow \infty$.

We now consider Riabounchinsky type flows in order to solve (2.15).

\subsection{Solution of the type $\psi(x, y)=y \xi(x)$}

In order to obtain another class of solution of (2.19) we substitute

$$
\psi(x, y)=y \xi(x)
$$

into (2.19) and get the following equation

$$
\rho\left[\xi \xi^{\prime \prime \prime}-\xi^{\prime} \xi^{\prime \prime}\right]=\mu \xi^{\mathrm{IV}}+\alpha_{1}\left[\xi \xi^{\mathrm{V}}-\xi^{\prime} \xi^{\mathrm{IV}}\right]-\left(H+\frac{\mu}{K}\right) \xi^{\prime \prime},
$$

where $\xi(x)$ is an arbitrary function of $x$, primes denote the derivative with respect to $x$.

Integrating (3.2.2) once and equating the constant of integration equal to zero we obtain

$$
\mu \xi^{\prime \prime \prime}+\rho\left[\left(\xi^{\prime 2}-\xi \xi^{\prime \prime}\right)\right]+\alpha_{1}\left[\left(\xi \xi^{\mathrm{IV}}-2 \xi^{\prime} \xi^{\prime \prime \prime}+\xi^{\prime \prime 2}\right)\right]-\left(H+\frac{\mu}{K}\right) \xi^{\prime}=0 .
$$

For the solution of the above equation we write

$$
\xi(x)=\delta\left(1+\lambda \mathrm{e}^{\sigma x}\right)
$$

in which $\delta, \sigma$ and $\lambda$ are arbitrary real constants. Making use of (3.2.4) into (3.2.3) we have

$$
\delta=\frac{1}{\rho-\alpha_{1} \sigma^{2}}\left[\mu \sigma-\frac{1}{\sigma}\left(H+\frac{\mu}{K}\right)\right]
$$

and thus from (3.2.1)

$$
\psi(x, y)=\frac{1}{\rho-\alpha_{1} \sigma^{2}}\left[\mu \sigma-\frac{1}{\sigma}\left(H+\frac{\mu}{K}\right)\right] y\left(1+\lambda \mathrm{e}^{\sigma x}\right) .
$$




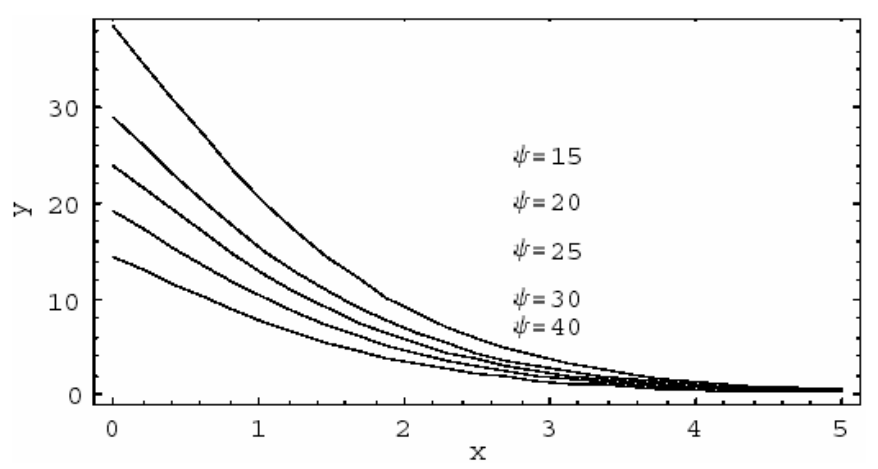

Figure 6. Streamline flow pattern for $\psi(x, y)=\frac{1}{\rho-\alpha_{1} \sigma^{2}}\left[\mu \sigma-\frac{1}{\sigma}\left(H+\frac{\mu}{K}\right)\right] y(1+$ $\left.\lambda \mathrm{e}^{\sigma x}\right)$.

The velocity components (2.18) and the pressure field (2.15) become

$$
\begin{aligned}
u= & \frac{1}{\rho-\alpha_{1} \sigma^{2}}\left[\mu \sigma-\frac{1}{\sigma}\left(H+\frac{\mu}{K}\right)\right]\left(1+\lambda \mathrm{e}^{\sigma x}\right), \\
v= & \frac{-y}{\rho-\alpha_{1} \sigma^{2}}\left[\mu \sigma-\frac{1}{\sigma}\left(H+\frac{\mu}{K}\right)\right] \sigma \lambda \mathrm{e}^{\sigma x} . \\
p= & p_{1}+\mu \sigma \bar{a}\left(1-\frac{\sigma^{2} y^{2}}{2}\right) \lambda \mathrm{e}^{\sigma x}-\frac{1}{2} \rho\left[\bar{a}^{2}\left(1-\lambda^{2} \mathrm{e}^{2 \sigma x}\right)\right] \\
& +\alpha_{1}\left[\bar{a}^{2} \sigma^{2} \lambda \mathrm{e}^{\sigma x}+\bar{a}^{2} \sigma^{2} \lambda^{2}\left(3+\frac{\sigma^{2} y^{2}}{2}\right) \mathrm{e}^{2 \sigma x}\right],
\end{aligned}
$$

where $p_{1}$ is the reference pressure and

$$
\bar{a}=\frac{1}{\rho-\alpha_{1} \sigma^{2}}\left[\mu \sigma-\frac{1}{\sigma}\left(H+\frac{\mu}{K}\right)\right] .
$$

The streamline flow for $\psi=\Omega_{4}$ is given by the functional form

$$
y=\frac{\Omega_{4}}{\left(1+\lambda \mathrm{e}^{\sigma x}\right) \varepsilon},
$$

where

$$
\varepsilon=\frac{1}{1-\Lambda \sigma^{2}}\left[\nu \sigma-\frac{1}{\sigma}\left(\chi+\frac{v}{K}\right)\right] .
$$

Figure 6 shows the streamlines for $\sigma=\lambda=1, \mu / \rho=0.5, \alpha_{1} / \rho=0.1, K=15, N=$ $0, \psi=15,20,25,30,40$.

\subsection{Solutions of the type $\psi(x, y)=y \xi(x)+\eta(x)$}

Inserting

$$
\psi(x, y)=y \xi(x)+\eta(x)
$$


in (2.19) we obtain the following equation

$$
\begin{aligned}
- & \rho\left[y\left(\xi^{\prime} \xi^{\prime \prime}-\xi \xi^{\prime \prime \prime}\right)+\left(\eta^{\prime} \xi^{\prime \prime}-\xi \eta^{\prime \prime \prime}\right)\right] \\
= & \mu\left(y \xi^{\mathrm{IV}}+\eta^{\mathrm{IV}}\right)-\alpha_{1}\left[y\left(\xi^{\prime} \xi^{\mathrm{IV}}-\xi \xi^{\mathrm{V}}\right)+\left(\eta^{\prime} \xi^{\mathrm{IV}}-\xi \eta^{\mathrm{V}}\right)\right] \\
& -\left(H+\frac{\mu}{K}\right)\left(y \xi^{\prime \prime}+\eta^{\prime \prime}\right) .
\end{aligned}
$$

From the above equation, we have

$$
\rho\left[\xi^{\prime} \xi^{\prime \prime}-\xi \xi^{\prime \prime \prime}\right]+\mu \xi^{\mathrm{IV}}-\alpha_{1}\left[\xi^{\prime} \xi^{\mathrm{IV}}-\xi \xi^{\mathrm{V}}\right]-\left(H+\frac{\mu}{K}\right) \xi^{\prime \prime}=0
$$

and

$$
\rho\left[\eta^{\prime} \xi^{\prime \prime}-\xi \eta^{\prime \prime \prime}\right]+\mu \eta^{\mathrm{IV}}-\alpha_{1}\left[\eta^{\prime} \xi^{\mathrm{IV}}-\xi \eta^{\mathrm{V}}\right]-\left(H+\frac{\mu}{K}\right) \eta^{\prime \prime}=0
$$

where $\xi(x)$ and $\eta(x)$ are arbitrary functions of its arguments. Integrating (3.3.3) and (3.3.4) and then taking the constants of integration equal to zero we have

$$
\begin{aligned}
& \mu \xi^{\prime \prime \prime}+\rho\left[\xi^{\prime 2}-\xi \xi^{\prime \prime}\right]-\alpha_{1}\left[\left(-\xi \xi^{\mathrm{IV}}+2 \xi^{\prime} \xi^{\prime \prime \prime}-\xi^{\prime \prime 2}\right)\right]-\left(H+\frac{\mu}{K}\right) \xi^{\prime}=0 \\
& \mu \eta^{\prime \prime \prime}+\rho\left[\eta^{\prime} \xi^{\prime}-\xi \eta^{\prime \prime}\right]-\alpha_{1}\left[\begin{array}{c}
\xi^{\prime} \eta^{\prime \prime \prime}-\xi \eta^{\mathrm{IV}} \\
+\eta^{\prime} \xi^{\prime \prime \prime}-\eta^{\prime \prime} \xi^{\prime \prime}
\end{array}\right]-\left(H+\frac{\mu}{K}\right) \eta^{\prime}=0
\end{aligned}
$$

We note that (3.3.5) is similar to (3.2.3). Its solution is given in (3.2.4). Substituting (3.2.4) into (3.3.6) we have

$$
\begin{gathered}
\alpha_{1} A\left(1+\lambda \mathrm{e}^{\sigma x}\right) \eta^{\mathrm{IV}}+\left(\mu-\alpha_{1} A \lambda \sigma \mathrm{e}^{\sigma x}\right) \eta^{\prime \prime \prime}+A\left[\left(\alpha_{1} \sigma^{2}-\rho\right) \lambda \mathrm{e}^{\sigma x}-\rho\right] \eta^{\prime \prime} \\
=-\left[\left(\rho-\alpha_{1} \sigma^{2}\right) A \lambda \sigma \mathrm{e}^{\sigma x}-\left(H+\frac{\mu}{K}\right)\right] \eta^{\prime}
\end{gathered}
$$

where

$$
A=\frac{1}{\rho-\alpha_{1} \sigma^{2}}\left[\mu \sigma-\frac{1}{\sigma}\left(H+\frac{\mu}{K}\right)\right] .
$$

We note that it is not easy to obtain the general solution of (3.3.7). In order to find its solution we consider the following special cases:

Case 1. $\alpha_{1} \neq 0, \sigma=1, \lambda=0$

Equation (3.3.7) reduces to

$$
\alpha_{1} A_{1} \eta^{\mathrm{IV}}+\mu \eta^{\prime \prime \prime}-\rho A_{1} \eta^{\prime \prime}-\left(H+\frac{\mu}{K}\right) \eta^{\prime}=0 .
$$

We see that (3.3.8) is of fifth order and in order to solve it we reduce its order by putting $\eta^{\prime}=\bar{A}(x)$ such that (3.3.8) becomes

$$
\alpha_{1} A_{1} \bar{A}^{\prime \prime \prime}+\mu \bar{A}^{\prime \prime}-\rho A_{1} \bar{A}^{\prime}-\left(H+\frac{\mu}{K}\right) \bar{A}=0 .
$$


On substituting $\bar{A}(x)=\widehat{P}(x) \mathrm{e}^{x},(3.3 .9)$ takes the form

$$
\alpha_{1}\left(3 \widehat{P}^{\prime}+3 \widehat{P}^{\prime \prime}+\widehat{P}^{\prime \prime \prime}\right) \mathrm{e}^{x}+\frac{\mu}{A_{1}}\left(2 \widehat{P}^{\prime}+\widehat{P}^{\prime \prime}\right) \mathrm{e}^{x}-\rho \widehat{P}^{\prime} \mathrm{e}^{x}=0 .
$$

Finally, $\widehat{P}^{\prime}(x)=R(x)$ converts (3.3.10) into a second-order differential equation

$$
\alpha_{1} R^{\prime \prime}+\left(\mu / A_{1}+3 \alpha_{1}\right) R^{\prime}+\left(3 \alpha_{1}-\rho+2 \mu / A_{1}\right) R=0 .
$$

The solution of the above equation is

$$
R(x)=A_{3} \exp \left(\frac{-c-\sqrt{c^{2}-4 d}}{2}\right) x+A_{4} \exp \left(\frac{-c+\sqrt{c^{2}-4 d}}{2}\right) x,
$$

where $A_{3}$ and $A_{4}$ are arbitrary constants and

$$
\begin{aligned}
& c=\frac{3 \alpha_{1} A_{1}+\mu}{\alpha_{1} A_{1}}, \quad d=\frac{\left(3 \alpha_{1}-\rho\right) A_{1}+2 \mu}{\alpha_{1} A_{1}} \\
& A_{1}=\frac{1}{\rho-\alpha_{1}}\left[\mu\left(1-\frac{1}{K}\right)-H\right] .
\end{aligned}
$$

In order to find $\eta(x)$ we make backward substitutions and finally obtain the form

$$
\eta(x)=\frac{A_{3}}{m_{1}\left(1+m_{1}\right)} \mathrm{e}^{\left(1+m_{1}\right) x}+\frac{A_{4}}{m_{2}\left(1+m_{2}\right)} \mathrm{e}^{\left(1+m_{2}\right) x}+A_{5} \mathrm{e}^{x}+A_{6} x,
$$

where $A_{i}(i=5,6)$ are constants of integration and

$$
m_{1}=\frac{-c-\sqrt{c^{2}-4 d}}{2}, \quad m_{2}=\frac{-c+\sqrt{c^{2}-4 d}}{2} .
$$

From (3.2.4), (3.3.1) and (3.3.13) we get

$$
\begin{aligned}
\psi(x, y)= & \frac{y}{\rho-\alpha_{1}}\left[\mu\left(1-\frac{1}{K}\right)-H\right]+A_{5} \mathrm{e}^{x}+A_{6} \\
& +\frac{A_{3}}{m_{1}\left(1+m_{1}\right)} \mathrm{e}^{\left(1+m_{1}\right) x}+\frac{A_{4}}{m_{2}\left(1+m_{2}\right)} \mathrm{e}^{\left(1+m_{2}\right) x}
\end{aligned}
$$

The velocity components and pressure field are

$$
\begin{aligned}
& u=\frac{1}{\rho-\alpha_{1}}\left[\mu\left(1-\frac{1}{K}\right)-H\right], \\
& v=-\left[\frac{A_{3}}{m_{1}} \mathrm{e}^{\left(1+m_{1}\right) x}+\frac{A_{4}}{m_{2}} \mathrm{e}^{\left(1+m_{2}\right) x}+A_{5} \mathrm{e}^{x}\right] \text {, } \\
& p=p_{2}-\frac{1}{2} \rho\left[A_{1}^{2}\right] \\
& +\alpha_{1}\left[\begin{array}{c}
\frac{A_{3}^{2}}{m_{1}^{2}} \mathrm{e}^{2\left(1+m_{1}\right) x}+\frac{2 A_{3} A_{4}}{m_{1} m_{2}} \mathrm{e}^{\left(2+m_{1}+m_{2}\right) x}+\frac{A_{4}^{2}}{m_{2}^{2}} \mathrm{e}^{2\left(1+m_{2}\right) x}+A_{5}^{2} \mathrm{e}^{2 x} \\
+\frac{2 A_{3} A_{5}\left(2+3 m_{1}+m_{1}^{2}\right)}{\left(2+m_{1}\right) m_{1}} \mathrm{e}^{\left(2+m_{1}\right) x}+\frac{2 A_{4} A_{5}\left(2+3 m_{2}+m_{2}^{2}\right)}{\left(2+m_{2}\right) m_{2}} \mathrm{e}^{\left(2+m_{2}\right) x}
\end{array}\right],
\end{aligned}
$$

where $p_{2}$ is the reference pressure. 


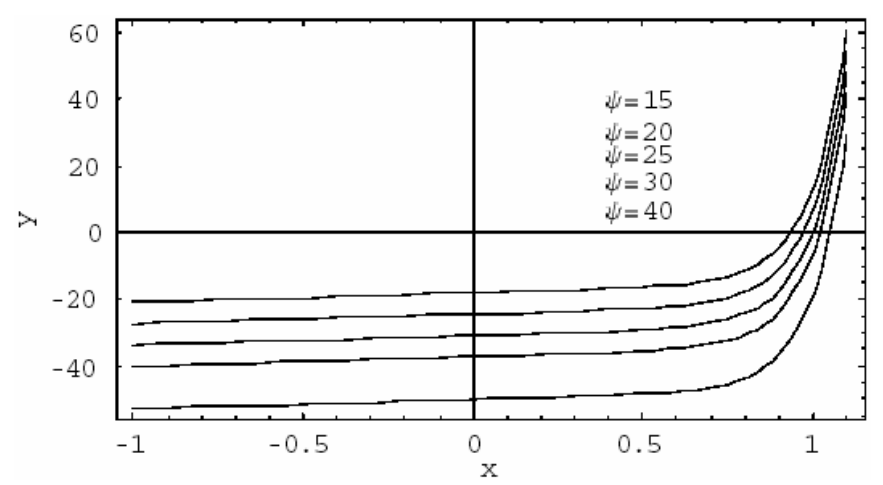

Figure 7. Streamline flow pattern for $\psi(x, y)=\frac{y}{\rho-\alpha_{1}}\left[\mu\left(1-\frac{1}{K}\right)-H\right]+A_{5} \mathrm{e}^{x}$ $+A_{6}+\frac{A_{3}}{m_{1}\left(1+m_{1}\right)} \mathrm{e}^{\left(1+m_{1}\right) x}+\frac{A_{4}}{m_{2}\left(1+m_{2}\right)} \mathrm{e}^{\left(1+m_{2}\right) x}$.

The streamline for $\psi=\Omega_{5}$ is given by the functional form

$$
y=-\frac{1}{\varepsilon_{1}}\left[\begin{array}{c}
-\Omega_{5}+\frac{A_{3}}{m_{1}\left(1+m_{1}\right)^{2}} \mathrm{e}^{\left(1+m_{1}\right) x} \\
+\frac{A_{4}}{m_{2}\left(1+m_{2}\right)^{2}} \mathrm{e}^{\left(1+m_{2}\right) x}+A_{5} \mathrm{e}^{x}+A_{6} x
\end{array}\right],
$$

where

$$
\varepsilon_{1}=\frac{1}{1-\Lambda}\left[v\left(1-\frac{1}{K}\right)-\chi\right]
$$

Streamline pattern is plotted in figure 7 for $\sigma=\lambda=1, \mu / \rho=0.5, \alpha_{1} / \rho=0.1, K=0.5$, $N=0, \varphi=0.05, A 3=A 4=A 5=A 6=1, \psi=15,20,25,30,40$.

Case 2. $\alpha_{1} \neq 0, \sigma=1, \lambda \neq 0$

Equation (3.3.7) reduces to

$$
\begin{aligned}
& \alpha_{1} A_{1}\left(1+\lambda \mathrm{e}^{x}\right) \eta^{\mathrm{IV}}+\left(\mu-\alpha_{1} A_{1} \lambda \mathrm{e}^{x}\right) \eta^{\prime \prime \prime}+A_{1}\left[\left(\alpha_{1}-\rho\right) \lambda \mathrm{e}^{x}-\rho\right] \eta^{\prime \prime} \\
& =-\left[\left(\rho-\alpha_{1}\right) A_{1} \lambda \mathrm{e}^{x}-\left(H+\frac{\mu}{K}\right)\right] \eta^{\prime}
\end{aligned}
$$

To obtain the solution of (3.3.19) we try to reduce its order. For this purpose we put $\eta^{\prime}=\widehat{A}(x)$ which leaves (3.3.19) into a form which is one order less, that is

$$
\begin{gathered}
\alpha_{1}\left(1+\lambda \mathrm{e}^{x}\right) \widehat{A}^{\prime \prime \prime}+\left(\mu / A_{1}-\alpha_{1} \lambda \mathrm{e}^{x}\right) \widehat{A}^{\prime \prime}+\left[\left(\alpha_{1}-\rho\right) \lambda \mathrm{e}^{x}-\rho\right] \widehat{A}^{\prime} \\
=-\left[\left(\rho-\alpha_{1}\right) \lambda \mathrm{e}^{x}-\frac{1}{A_{1}}\left(H+\frac{\mu}{K}\right)\right] \widehat{A}=0 .
\end{gathered}
$$

Now substituting $\widehat{A}(x)=\bar{P}(x) \mathrm{e}^{x}$ in (3.3.20) and then $\bar{P}^{\prime}(x)=R(x)$ into the resulting expression, we get

$$
\begin{gathered}
\alpha_{1}\left(1+\lambda \mathrm{e}^{x}\right) R^{\prime \prime}+\left[\frac{K \mu\left(\rho-\alpha_{1}\right)}{K(\mu-H)-\mu}+\alpha_{1}\left(3+2 \lambda \mathrm{e}^{x}\right)\right] R^{\prime} \\
=-\left[\frac{2 K \mu\left(\rho-\alpha_{1}\right)}{K(\mu-H)-\mu}+\left(2 \alpha_{1}-\rho\right)\left(1+\lambda \mathrm{e}^{x}\right)\right] R,
\end{gathered}
$$

where we have taken the constant of integration equal to zero. 
Subcase 1. The solution of (3.3.21) for $\lambda=0$ is given by

$$
\begin{aligned}
R(x)= & C_{1} \exp \left(\frac{-X_{1}-\sqrt{X_{1}^{2}-4 X_{2}}}{2}\right) x \\
& +C_{2} \exp \left(\frac{-X_{1}+\sqrt{X_{1}^{2}-4 X_{2}}}{2}\right) x,
\end{aligned}
$$

where $C_{1}$ and $C_{2}$ are arbitrary constants and

$$
X_{1}=\frac{K \mu\left(\rho-\alpha_{1}\right)}{\alpha_{1}[K(\mu-H)-\mu]}+3, X_{2}=\frac{2 K \mu\left(\rho-\alpha_{1}\right)}{\alpha_{1}[K(\mu-H)-\mu]}+\frac{3 \alpha_{1}-\rho}{\alpha_{1}} .
$$

The backward substitution gives the value of $\eta(x)$ as

$$
\eta(x)=\frac{C_{1}}{\bar{m}_{1}\left(1+\bar{m}_{1}\right)} \mathrm{e}^{\left(1+\bar{m}_{1}\right) x}+\frac{C_{2}}{\bar{m}_{2}\left(1+\bar{m}_{2}\right)} \mathrm{e}^{\left(1+\bar{m}_{2}\right) x}+C_{3} \mathrm{e}^{x}+C_{4},
$$

where $C_{i}(i=1,2,3,4)$ are constants of integration and

$$
\bar{m}_{1}=\frac{-X_{1}-\sqrt{X_{1}^{2}-4 X_{2}}}{2}, \quad \bar{m}_{2}=\frac{-X_{1}+\sqrt{X_{1}^{2}-4 X_{2}}}{2} .
$$

The stream function, the velocity components and the pressure field in this case are respectively given as

$$
\begin{aligned}
& \psi(x, y)=\frac{y}{\rho-\alpha_{1}}\left[\mu\left(1-\frac{1}{K}\right)-H\right]+C_{3} \mathrm{e}^{x}+C_{4} \\
& +\frac{C_{1}}{\bar{m}_{1}\left(1+\bar{m}_{1}\right)} \mathrm{e}^{\left(1+\bar{m}_{1}\right) x}+\frac{C_{2}}{\bar{m}_{2}\left(1+\bar{m}_{2}\right)} \mathrm{e}^{\left(1+\bar{m}_{2}\right) x}, \\
& u=\frac{1}{\rho-\alpha_{1}}\left[\mu\left(1-\frac{1}{K}\right)-H\right] \text {, } \\
& v=-\left[\frac{C_{1}}{\bar{m}_{1}} \mathrm{e}^{\left(1+\bar{m}_{1}\right) x}+\frac{C_{2}}{\bar{m}_{2}} \mathrm{e}^{\left(1+\bar{m}_{2}\right) x}+C_{3} \mathrm{e}^{x}\right] \text {, } \\
& p=p_{3}-\frac{1}{2} \rho\left[A_{1}^{2}\right] \\
& +\alpha_{1}\left[\begin{array}{l}
\frac{C_{1}^{2}}{\bar{m}_{1}^{2}} \mathrm{e}^{2\left(1+\bar{m}_{1}\right) x}+\frac{2 C_{1} C_{2}}{\bar{m}_{1} \bar{m}_{2}} \mathrm{e}^{\left(2+\bar{m}_{1}+\bar{m}_{2}\right) x}+\frac{C_{2}^{2}}{\bar{m}_{2}^{2}} \mathrm{e}^{2\left(1+\bar{m}_{2}\right) x}+C_{3}^{2} \mathrm{e}^{2 x} \\
+\frac{2 C_{1} C_{3}\left(2+3 \bar{m}_{1}+\bar{m}_{1}^{2}\right)}{\left(2+\bar{m}_{1}\right) \bar{m}_{1}} \mathrm{e}^{\left(2+\bar{m}_{1}\right) x}+\frac{2 C_{2} C_{3}\left(2+3 \bar{m}_{2}+\bar{m}_{2}^{2}\right)}{\left(2+\bar{m}_{2}\right) \bar{m}_{2}} \mathrm{e}^{\left(2+\bar{m}_{2}\right) x}
\end{array}\right],
\end{aligned}
$$

where $p_{3}$ is the reference pressure and the streamline for $\psi=\Omega_{6}$ is given by the functional form

$$
y=-\frac{1}{\varepsilon_{1}}\left[\begin{array}{c}
-\Omega_{6}+\frac{C_{1}}{\bar{m}_{1}\left(1+\bar{m}_{1}\right)^{2}} \mathrm{e}^{\left(1+\bar{m}_{1}\right) x} \\
+\frac{C_{2}}{\bar{m}_{2}\left(1+\bar{m}_{2}\right)^{2}} \mathrm{e}^{\left(1+\bar{m}_{2}\right) x}+C_{3} \mathrm{e}^{x}+C_{4}
\end{array}\right] .
$$

Streamline pattern is plotted in figure 8 for $\sigma=\lambda=1, \mu / \rho=0.5, \alpha_{1} / \rho=0.1, K=1.8$, $N=1, \varphi=1, C_{1}=C_{2}=C_{3}=C_{4}=1, \psi=15,20,25,30,40$. 


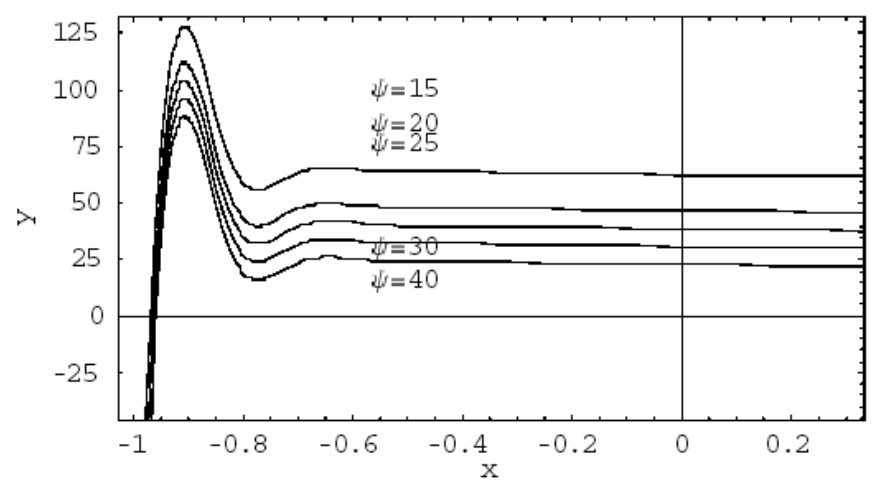

Figure 8. Streamline flow pattern for $\psi(x, y)=\frac{y}{\rho-\alpha_{1}}\left[\mu\left(1-\frac{1}{K}\right)-H\right]+C_{3} \mathrm{e}^{x}+$ $C_{4}+\frac{C_{1}}{\bar{m}_{1}\left(1+\bar{m}_{1}\right)} \mathrm{e}^{\left(1+\bar{m}_{1}\right) x}+\frac{C_{2}}{\bar{m}_{2}\left(1+\bar{m}_{2}\right)} \mathrm{e}^{\left(1+\bar{m}_{2}\right) x}$.

Subcase 2.(General case). We now try to obtain the solution of (3.3.21) for $\lambda \neq 0$. Equation (3.3.21) may be written as

$$
\left(1+\lambda \mathrm{e}^{x}\right) R^{\prime \prime}+\left[X_{1}+2 \lambda \mathrm{e}^{x}\right] R^{\prime}+\left[X_{2}+X_{3} \lambda \mathrm{e}^{x}\right] R=0 .
$$

In order to solve (3.3.30) we put $\theta=\mathrm{e}^{x}$ to get the following equation

$$
(1+\lambda \theta) \theta^{2} R^{\prime \prime}+\left(1+X_{1}+2 \lambda \theta\right) \theta R^{\prime}+\left(X_{2}+X_{3} \lambda \theta\right) R=0,
$$

where differentiation is with respect to $\theta$. The solution for (3.3.31) is obtained through Mathematica and is given by

$$
\begin{aligned}
R(\theta)= & \theta^{1 / 2}\left(-X_{1}-\sqrt{X_{1}^{2}-4 X_{2}}\right) \\
& \times\left[\begin{array}{c}
\left.C_{5} \theta \sqrt{X_{1}^{2}-4 X_{2}} 2 F 1\left\{\frac{1}{2} \Phi_{1}, \frac{1}{2} \Phi_{2}, 1+\sqrt{X_{1}^{2}-4 X_{2}},-\theta \lambda\right\}\right] \\
+C_{1} 2 F 1\left\{\frac{1}{2} \Phi_{3}, \frac{1}{2} \Phi_{4}, 1-\sqrt{X_{1}^{2}-4 X_{2}},-\theta \lambda\right\}
\end{array}\right],
\end{aligned}
$$

where

$$
\begin{aligned}
& \Phi_{1}=2-X_{1}+\sqrt{X_{1}^{2}-4 X_{2}}-2 \sqrt{1-X_{3}}, \\
& \Phi_{2}=2-X_{1}+\sqrt{X_{1}^{2}-4 X_{2}}+2 \sqrt{1-X_{3}}, \\
& \Phi_{3}=2-X_{1}-\sqrt{X_{1}^{2}-4 X_{2}}-2 \sqrt{1-X_{3}}, \\
& \Phi_{4}=2-X_{1}-\sqrt{X_{1}^{2}-4 X_{2}}+2 \sqrt{1-X_{3}},
\end{aligned}
$$

and ${ }_{2} F_{1}$ is the hypergeometric function defined in Appendix A. The stream function, velocity components and the pressure field can be obtained through the definitions of ${ }_{2} F_{1}[a, b, c, z]$. 


\section{Concluding remarks}

In this paper, the exact solutions of non-linear equations governing the flow for a secondgrade fluid in a porous medium are obtained by assuming different forms of the stream function (already used by various authors in different situations), in presence of a strong magnetic field. The expressions for velocity profile, streamline and pressure distribution are constructed in each case. Our result indicates that velocity, stream function and pressure are strongly dependent upon the material parameter $\alpha_{1}$ of the second-grade fluid. It is shown through graphs that increase in $\alpha_{1}$ leads to decrease in velocity and decrease in $\alpha_{1}$ leads to increase in velocity (see figures 4 and 5). Also, the present analysis is more general and several results of various authors (as already mentioned in the text) can be recovered in the limiting cases.

\section{Appendix A}

Hypergeometric ${ }_{2} F_{1}[a, b, c, z]$ is the hypergeometric function ${ }_{2} F_{1}[a, b ; c ; z]$ and is the special case of the generalized hypergeometric function ${ }_{p} F_{q}[\mathbf{a} ; \mathbf{b} ; z]$ for $p=1$ and $q=1$.

Hypergeometric function has the following properties:

1. The ${ }_{2} F_{1}$ function has the series expansion ${ }_{2} F_{1}[a, b ; c ; z]=\sum_{k=0}^{\infty} \frac{(a)_{k}(b)_{k}}{(c)_{k}} \frac{z^{k}}{k !}$.

2. Hypergeometric ${ }_{2} F_{1}[a, b, c, z]$ has a branch cut discontinuity in the complex $z$-plane running from 1 to $\infty$.

\section{References}

[1] Anderson H I and Holmedal L E, Start-up flow in a porous medium channel, Acta Mechanica 113 (1995) 155

[2] Bear J, Dynamics of fluids in porous media (New York: Elsevier) (1972)

[3] Benharbit A M and Siddiqui A M, Certain solutions of the equations of the planar motion of a second-grade fluid for steady and unsteady cases, Acta Mech. 94 (1992) 85

[4] Berker R, Integration des equations du mouvemont d'un fluide visqueux incompressible, Handbuk der Physik VII (Berlin: Springer) (1963)

[5] Brinkman H C, A calculation of the viscous force extended by a flowing fluid on a dense swarm of particles, Appl. Sci. Res. A1 (1947) 27

[6] Cowling T G, Magnetohydrodynamics (New York: Interscience) (1957) p. 101

[7] Dunn J E and Fosdick R L, Thermodynamics, stability and boundedness of fluids of complexity 2 and fluids of second grade, Arch. Rat. Mech. Anal. 56 (1974) 191

[8] Fosdick R L and Rajagopal K R, Anomalous features in the model of second order fluids, Arch. Ration. Mech. Anal. 70 (1979) 145

[9] Hossain M A, Effect of Hall current on unsteady hydromagnetic free convection flow near an infinite vertical porous plate, J. Phys. Soc. Jpn. 55(7) (1986) 2183-2190

[10] Hossain M A and Mohammad K, Effect of Hall current on hydromagnetic free convection flow near an accelerated porous plate, Jpn. J. Appl. Soc. 27(8) (1988) 1531-1535

[11] Hossain M A and Rashid R I M A , Effect of Hall current on hydromagnetic free convection flow near along a porous flat plate with mass transfer, J. Phys. Soc. Jpn. 56(7) (1987) 97-104

[12] Kalony P N and Huschilt K, Semi inverse solutions of a non-Newtonian fluid, Int. J. Non-Linear Mech. 19 (1984) 373 
[13] Kaviany M, Laminar flow through a porous channel bounded by isothermal parallel plates, Int. J. Heat Mass Transfer 28 (1985) 851

[14] Kodadadi J M, Oscillatory fluid flow through a porous medium channel bounded by two impermeable parallel plates, in: International Symposium on Nonsteady Fluid Dynamics, FED, vol. 92, pp. 243, ASME Fluids Engineering Spring Conference, Toronto, Ontario, Canada (1990)

[15] Kodadadi J M, Oscillatory fluid flow through a porous medium channel bounded by two impermeable parallel plates, ASME J. Fluids Eng. 113 (1991) 509

[16] Labropulu F, Exact solutions of non-Newtonian fluid flows with prescribed vorticity, Acta Mech. 141 (2000) 11

[17] Nemenyi P N, Recent developments in inverse and semi-inverse methods in the mechanics of continua, Advances Appl. Mech. (New York) (1951) p. 2

[18] Pop I, The effect of Hall currents on hydromagnetic flow near an accelerated plate, $J$. Math. Phys. Sci. 5 (1971) 375-385

[19] Rajagopal K R, On the boundary conditions for fluids of the differential type, in: A Sequeira (ed.) Navier-Stokes equations and related non-linear problems (New York: Plenum Press) (1995) p. 273

[20] Ram P C, Hall effects on free convection flow and mass transfer through a porous medium, Waerme-Stoffubertrag 22 (1988) 223-225

[21] Raptis A and Ram P C, Effects of Hall current and rotation, Astrophys. Space Sci. 106 (1984) 257-264

[22] Rivlin R S and Ericksen J L, Stress deformation relations for isotropic materials, J. Rat. Mech. Anal. 4 (1955) 323

[23] Sato $\mathrm{H}$, The Hall effects in the viscous flow of ionized gas between parallel plates under transverse magnetic field, J. Phys. Soc. Jpn. 16 (1961) 1427-1433

[24] Sherman A and Sutton G W, Engineering magnetohydrodynamics (New York: McGrawHill) (1965)

[25] Siddiqui A M and Kalony P N, Certain inverse solutions of a non-Newtonian fluid, Int. J. Non-Linear Mech. 21 (1986) 459

[26] Siddiqui A M, Some more inverse solutions of a non-Newtonian fluid, Mech. Res. Commun. 17 (1990) 157

[27] Siddiqui A M, Mohyuddin M R, Hayat T and Asghar S, Some more inverse solutions for steady flows of a second-grade fluid, Arch. Mech. 55 (2003) 373

[28] Vafai $\mathrm{K}$ and Tien C L, Boundary and inertia effects on flow and heat transfer in porous media, Int. J. Heat Mass Transfer 24 (1981) 195 